\title{
Mid-Infrared Lasing in Lead Sulfide Subwavelength Wires on Silicon Supporting Information
}

\author{
Fan Fan, Zhicheng Liu, Minghua Sun, Patricia L. Nichols, Sunay Turkdogan, and C. Z. Ning* \\ School of Electrical, Computer and Energy Engineering, Arizona State University, Tempe, AZ 85287 \\ *Corresponding author: cning@asu.edu
}

\section{Internal Quantum Efficiency of PbS and Comparison with InAs}

To demonstrate the high luminesce efficiency of $\mathrm{PbS}$ material as we mentioned in the introductory part of the paper, we perform theoretical calculation of internal quantum efficiency $(\mathrm{QE})$ for $\mathrm{PbS}$ and compare it with a common III-V material with similar bandgap, InAs.

The internal quantum efficiency is defined as:

$$
\eta_{\mathrm{i}}=\frac{B n^{2}}{A n+B n^{2}+C n^{3}}
$$

where, $n$ is the carrier density, $A$ is the defect-related non-radiative (or Shockley-Reed-Hall) recombination rate, $B$ is the radiative recombination coefficient, and $C$ is the Auger recombination coefficient. The nonradiative recombination coefficient $A$ greatly depends on the crystal quality and a value of $5 \times 10^{7} \mathrm{~s}^{-1}$ is used for both $\mathrm{PbS}$ and InAs in calculation for a fair comparison. The Auger recombination coefficient has weak dependency to carrier-density and can be obtained from reference [S1], [S2]. The radiative recombination coefficient, however, is a non-linear function of carrier density, and has the form:

$$
B=\frac{R_{s p}}{n^{2}}
$$

where, $R_{s p}$ is the spontaneous emission rate. The calculation of the spontaneous emission rate involves solving semiconductor band structure. For simplicity, we here adopt the non-parabolic approximation [S3], [S4] for both $\mathrm{PbS}$ and InAs in their $E$ - $k$ relations, or:

$$
E(1+\alpha E)=\frac{\hbar^{2} k^{2}}{2 m_{e}}
$$

and the spontaneous emission rate is given by [S5]: 


$$
R_{s p}=\frac{n_{r} e^{2} \hbar \omega}{3 \pi \hbar^{2} \varepsilon_{0} m_{0}^{2} c^{3}}\left|\hat{\boldsymbol{e}} \cdot \overrightarrow{\boldsymbol{p}}_{c v}\right|^{2} \int_{E_{g}}^{\infty} N_{c v}(E) f_{e} f_{h} \cdot \mathcal{L}(E-\hbar \omega) d E
$$

where $n_{r}$ is the refractive index of the semiconductor, $\omega$ is the photon frequency, $m_{0}$ is the electron mass, $N_{c v}$ is the joint density of states and depend on band structure in general, as through the non-parabolic parameter here; $f_{\mathrm{e}}$ and $f_{h}$ are the electron and hole Fermi distribution, respectively, $\left|\hat{\boldsymbol{e}} \cdot \overrightarrow{\boldsymbol{p}}_{c v}\right|^{2}$ is the matrix element, and $\mathcal{L}(E-\hbar \omega)$ is the lineshap broadening function. The matrix element is acquired from Kane's model [S6], where:

$$
E_{p}=\frac{2\left|p_{c v}\right|^{2}}{m_{0}}
$$

and,

$$
\frac{E_{g}}{E_{p}}=\frac{m_{e}}{m_{0}}
$$
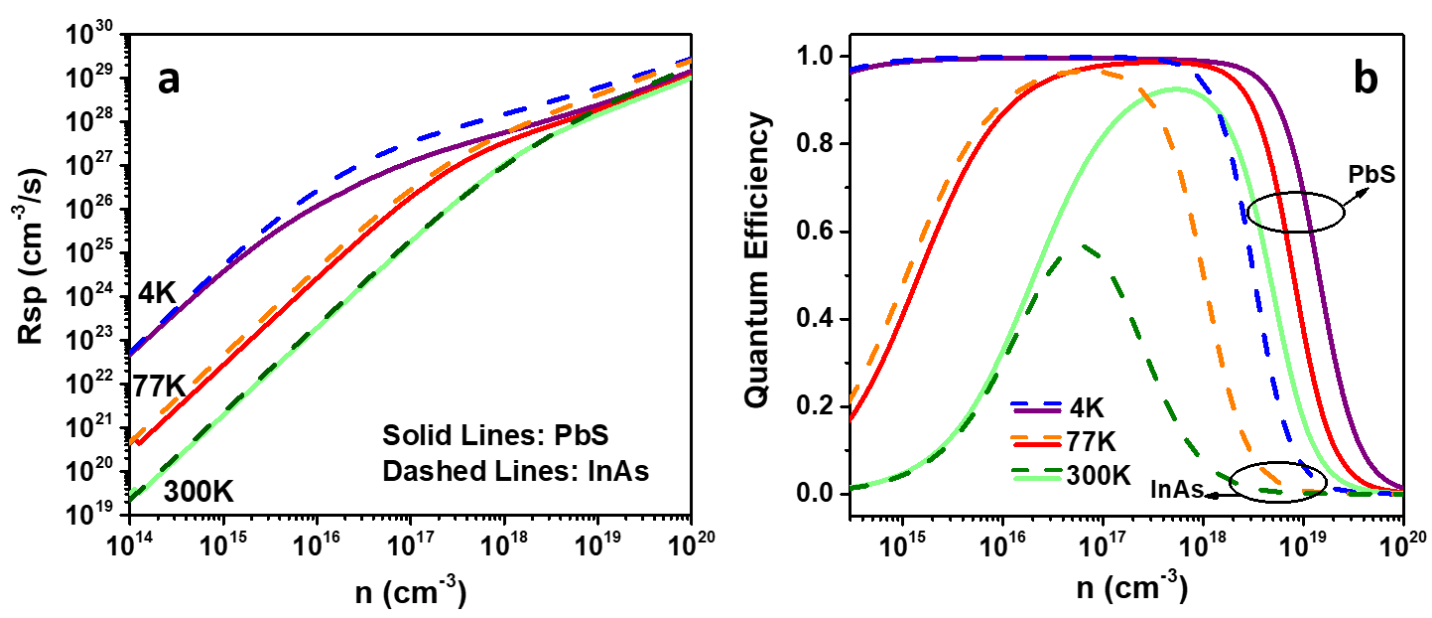

Figure S1. Calculation of internal QE. a) Calculation of spontaneous emission rate of $\mathrm{PbS}$ and InAs. b) Comparison of luminesce quantum efficiency of $\mathrm{PbS}$ and $\mathrm{InAs}$ at different temperature.

Figure S1 shows the calculated spontaneous emission rates and the internal QE. As can been noticed, the spontaneous emission rates of $\mathrm{PbS}$ and InAs are in the same orders of magnitude at different levels of carrier density in Fig. S1a, while the plot of QE can be differentiated into two different regimes in Fig. S1b. At low carrier density regime, the defect related non-radiative recombination dominates and the QE for $\mathrm{PbS}$ and InAs exhibits similar behavior. At high carrier density regime, however, the equivalent electron and hole masses of $\mathrm{PbS}$ lead to a reduced Auger recombination, resulting in a much higher QE than InAs, where the QE of InAs quickly dropped down close to zero when the carrier density is larger than $5 \times 10^{18} \mathrm{~cm}^{-3}$. 


\section{Effect of Pb-Rich Tips on Cavity Q-Factor}

$\mathrm{The} \mathrm{Pb}$-rich tips are result of self-catalyzed VLS growth and are present in all wires we grow. Sometimes the tips are broken off during sample preparation procedures. Wires with and without tips are both studied for lasing purpose. To examine the effects of $\mathrm{Pb}$-rich tips on cavity $\mathrm{Q}$-factor of $\mathrm{PbS}$ wires. We perform COMSOL Multiphysics ${ }^{\circledR} 3 \mathrm{D}$ simulation and compare the dominate mode with and without $\mathrm{Pb}$-rich tips attached to one end of the wire bodies. In the modal, the $\mathrm{PbS}$ wire is placed horizontally on a sapphire $\left(\mathrm{Al}_{2} \mathrm{O}_{3}\right)$ substrate similar to the characterization scenario in Fig.1c in the main text. A cubic geometry is adopted to represent the $\mathrm{Pb}$ tip shape instead of spherical geometry for the sake of simplicity of simulation. The dimensions of the wire are assumed to be $10 \mu \mathrm{m} \times 1.5 \mu \mathrm{m} \times 1.5 \mu \mathrm{m}$, while the value of refractive index of $\mathrm{PbS}$ and sapphire are 4.18 and 1.7 respectively at the wavelength range of around $3.2 \mu \mathrm{m}$. The dielectric constant of metal $\mathrm{Pb}$ is $\varepsilon_{\mathrm{r}}=-276.49+161.97 \mathrm{i}$ according to database [S7].
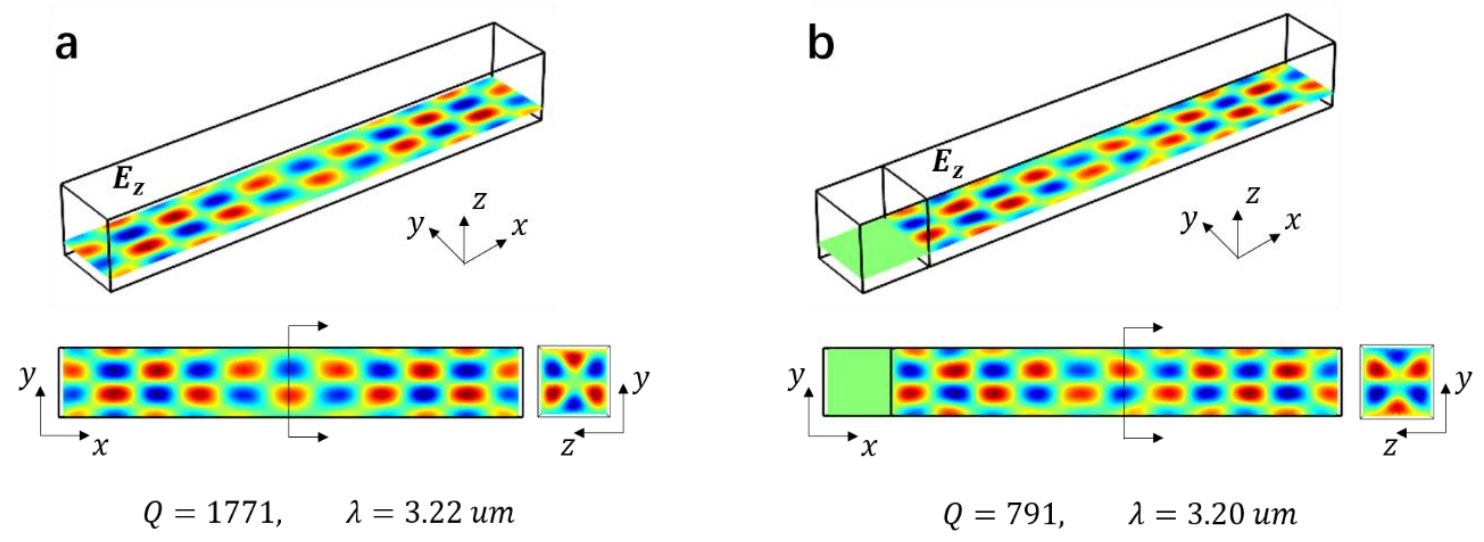

Figure S2. Simulated mode profile of a rectangular $\mathrm{PbS}$ wire on sapphire substrate and comparison of cavity Q-factor with (b) and without (a) Pb-rich tip. Top: schematic of the $\boldsymbol{E}_{\mathrm{z}}$ distribution. Bottom: the $\boldsymbol{E}_{\mathrm{Z}}$ profile inside the wire in $X Y$ and $Y Z$ plane.

Figure S2 shows the 3D simulation results. The calculated Q-factor in Fig. S2a is 1771 at the resonance wavelength of $3.22 \mu \mathrm{m}$ while the Q-factor of the wire attached by a Pb tip in Fig.S2b drops down to 791 at the resonance wavelength of $3.20 \mu \mathrm{m}$, mainly because of the metal absorption in $\mathrm{Pb}$. But the Q-factor is still larger enough for lasing. 


\section{Effects of Substrates on Cavity Q-Factor}

To quantitatively analyze the effects of silicon substrates on cavity Q-factor and understand the midinfrared lasing in as-grown wires, we perform 3D simulation in Fig. S3 and break down our analysis to two configurations and three different cases. Figure S3a shows the configuration of a transferred rectangular wire placed on a sapphire substrate. Figure S3b and S3c correspond to the configuration of a wire standing on silicon ( $\mathrm{Si}$ ) substrate in two separate scenarios: 1) standing on Si vertically (Fig.S3b); and 2) standing on $\mathrm{Si}$ at an angle of 45 degree (Fig.S3c). The wires are assumed to have the dimensions of $10 \mu \mathrm{m} \times 1.5 \mu \mathrm{m} \times 1.5 \mu \mathrm{m}$ in all three cases. As seen from the results, the mode of the rectangular wire in the sapphire substrate case has a Q of 1771, while due to the weak refractive index contrast between Si and $\mathrm{PbS}$, the $\mathrm{Q}$ of the wire on $\mathrm{Si}$ substrate in vertical case decreases dramatically down to 312 . On the contrary to Fig.S3b, the Q of the wire 45 degree on Si in Fig.S3c, however, has a significant increase to 989. We attribute the increased Q to the reduced transmission between $\mathrm{PbS}$ and Si interface at an angle, which explains the direct lasing from the as-grown wires observed in our experiment.
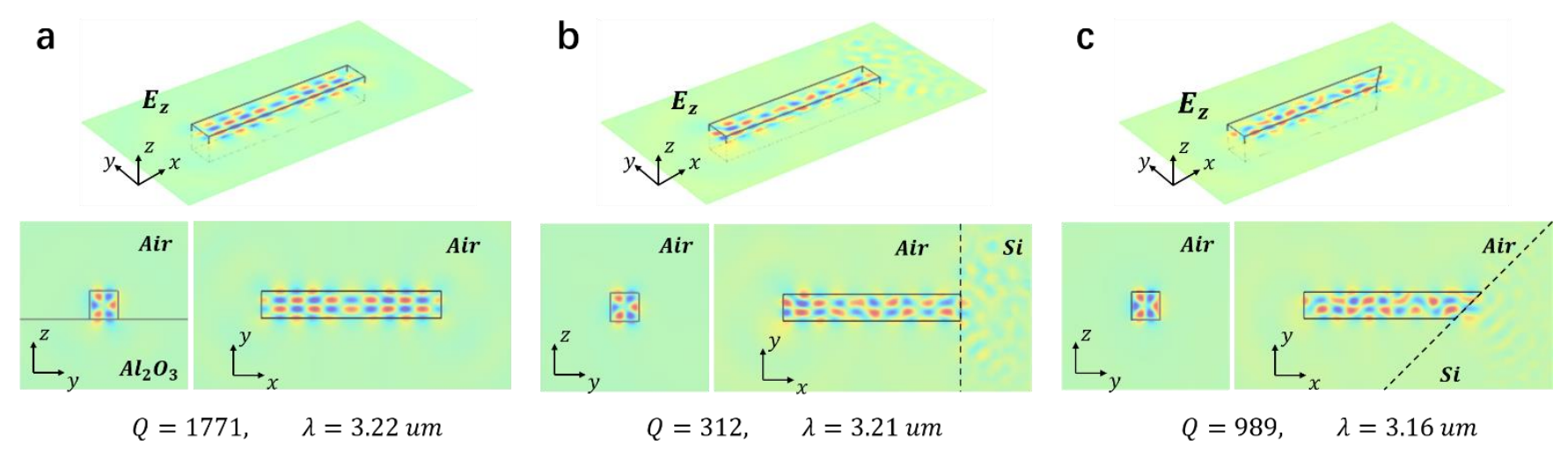

Figure S3. Simulated mode profile and cavity Q-factor for a rectangular wire on sapphire substrate (a), standing on Si substrate vertically (b), and standing on Si substrate at an angle of 45 degree (c). Top: schematic of the $\boldsymbol{E}_{\mathrm{z}}$ distribution. Bottom: the $\boldsymbol{E}_{\mathrm{z}}$ profile in $X Y$ and $Y Z$ plane.

It is worthy to point out that, we limit the length and diameter of the wire to be $10 \mu \mathrm{m}$ and $1.5 \mu \mathrm{m}$, and adopt a room temperature refractive index of 4.18 to facilitate mode identification with sufficient mode spacing. In reality, wires with longer length and larger diameters typically have a much higher Q, which help to overcome the loss and achieve lasing. 


\section{Statistics of Characterized PbS Wires}

A total of 32 wires were characterized in this work and 12 of them were able to demonstrate lasing. The wires were randomly selected from a dispersed sample and then transferred onto three different sapphire substrates for characterization. Table S1 is the summary of all the tested wires with lasing behavior observed (where the three samples discussed in the main text are labeled as A, B, C in the parenthesis). The yield can therefore be roughly estimated as $37.5 \%$.

Table S1. Summary of Characterized PbS Wires

\begin{tabular}{|c|c|c|c|c|}
\hline Sample \# & Lasing Type & Wavelength $(\mu \mathrm{m}) @ 77 \mathrm{~K}$ & Dimensions $\left(\mu \mathrm{m}^{3}\right)$ & Measured by \\
\hline$\# 1-5(A)$ & Multi-mode & $\lambda_{1}=3.1 ; \lambda_{2}=3.25$ & $13.1 \times 2.2 \times 0.7$ & SEM \\
\hline$\# 1-7$ & Single mode & $\lambda=2.99$ & $10.4 \times 1.6 \times \mathrm{NA}$ & Optical \\
\hline$\# 1-8$ (B) & Single mode & $\lambda=2.98$ & $7.4 \times 1.3 \times 1.2$ & SEM \\
\hline$\# 2-1$ & Single mode & $\lambda=3.19$ & $9.4 \times 3.2 \times \mathrm{NA}$ & Optical \\
\hline$\# 2-4$ & Multi-mode & $\begin{array}{l}\lambda_{1}=3.06 ; \lambda_{2}=3.13 ; \\
\lambda_{3}=3.17 ; \lambda_{4}=3.24\end{array}$ & $6.5 \times 2.3 \times 2.2$ & SEM \\
\hline$\# 2-5$ & Multi-mode & $\begin{array}{c}\lambda_{1}=3.04 ; \lambda_{2}=3.1 ; \\
\lambda_{3}=3.19 ; \lambda_{4}=3.23 ; \lambda_{5}=3.3\end{array}$ & $7.9 \times 2.0 \times 2.0$ & SEM \\
\hline$\# 2-6$ & Multi-mode & $\lambda_{1}=3.12 ; \lambda_{2}=3.22$ & $9.0 \times 1.3 \times \mathrm{NA}$ & Optical \\
\hline$\# 2-8$ (C) & Single mode & $\lambda=3.26$ & $6.7 \times 1.8 \times 1.4$ & SEM \\
\hline \#2-9 & Single mode & $\lambda=3.12$ & $6.6 \times 1.5 \times \mathrm{NA}$ & Optical \\
\hline \#2-11 & Multi-mode & $\begin{array}{l}\lambda_{1}=3.2 ; \lambda_{2}=3.25 \\
\lambda_{3}=3.27 ; \lambda_{4}=3.3\end{array}$ & $11.6 \times 3.0 \times \mathrm{NA}$ & Optical \\
\hline$\# 3-3$ & Multi-mode & $\lambda_{1}=3.16 ; \lambda_{2}=3.2$ & $16.3 \times 2.2 \times \mathrm{NA}$ & Optical \\
\hline$\# 3-6$ & Single mode & $\lambda=3.08$ & $10.4 \times 1.8 \times \mathrm{NA}$ & Optical \\
\hline
\end{tabular}

Lasing were not observed for the following wires: \#1-1, \#1-2, \#1-3, \#1-4, \#1-6, \#2-2, \#2-3, \#2-7, \#2-10, \#2-12, \#2-13, \#2-14, \#2-15, \#2-16, \#2-17, \#2-18, \#3-1, \#3-2, \#3-4, \#3-5. (Sizes were not recorded). 


\section{$\underline{\text { References }}$}

[S1] Peters, W. K., Cho, B., Hill, R. J., Courtney, T. L. \& Jonas, D. M. Band Filling Dynamics and Auger Recombination in Lead Sulfide Nanocrystals. Conference on Lasers and Electro-Optics (CLEO) TuE19 (2010)

[S2] Marchetti, S., Martinellil, M. \& Simili R. The Auger recombination coefficient in InAs and GaSb derived from the infrared dynamical plasma reflectivity. J. Phys: Condens. Matter 14, 3653-3656 (2002)

[S3] Mohammed, S. N. \& Abidi, S. T. H. Current, carrier concentration, Fermi energy, and related properties of binary compound polar semiconductors with nonparabolic energy bands. J. App. Phys. 60, 1384-1390 (1986)

[S4] InAs Electron Nonparabolicity, source: http://www.ioffe.ru/SVA/NSM/Semicond/InAs/bandstr.html (Retrieved Nov 30, 2019)

[S5] Singh, J. Optoelectronics: An Introduction to Materials and Devices. (McGraw-Hill Inc., 1996)

[S6] Chuang, S. L. Physics of Photonics Devices, $2^{\text {nd }}$ Edition. (John Wiley \& Sons Inc., 2009)

[S7] Refractive Index Database, source: https://refractiveindex.info/?shelf=main\&book=Pb\&page=Ordal, (Retrieved Nov 30, 2019). 